\title{
LA NOCIÓN DE LENGUAJE EN JACQUES LACAN: DEL SIGNO LINGUUÍSTICO EN SAUSSURE AL ALGORITMO SAUSSUREANO EN LACAN
}

\author{
FABIÁN BECERRA FUQUEN
}

Universidad de Santander-Udes, Colombia

(c) $\underset{\mathrm{BY}}{(0)}$ 


\title{
LA NOCIÓN DE LENGUAJE EN JACQUES LACAN: DEL SIGNO LINGÜÍSTICO EN SAUSSURE AL ALGORITMO SAUSSUREANO EN LACAN ${ }^{\star}$
}

\begin{abstract}
Resumen: el presente texto busca analizar la noción del lenguaje en Jacques Lacan teniendo como eje conductor el desarrollo que establece del algoritmo saussureano, siguiendo su antecedente a partir de la influencia que tuvo el signo lingüístico establecido por Ferdinand de Saussure. El signo lingüístico está compuesto por una estructura de propiedades mínimas: El significado y el significante, Lacan toma de éste el significante, lo extrae de la lingüística de Saussure para introducirlo al campo del psicoanálisis y formalizar la estructura del sujeto, de allí que se pueda advertir que el orden del significante da lugar al significado propio de la singularidad del sujeto, contrario al signo lingüístico que señala un campo más amplio de la significación, a nivel del grupo social.
\end{abstract}

Palabras clave: signo lingüístico, significante, estructura, sujeto, psicoanálisis.

\section{THE NOTION OF LANGUAGE IN JACQUES LACAN: FROM LINGUISTIC SIGN IN SAUSSURE TO LACAN SAUSSUREAN ALGORITHM}

\begin{abstract}
This paper seeks to analyze the notion of language in Jacques Lacan development from Lacan establishes the Saussurean algorithm, following its antecedent in the linguistic sign of Ferdinand de Saussure. The linguistic sign is composed of the signifier and the signified, Lacan takes the signifier, to introduce it to the field of psychoanalysis and formalize the structure of the subject, the signifier results in the signified that is the uniqueness of the subject, contrary to the linguistic sign that signals a broader significantion level social group field.
\end{abstract}

Keywords: Linguistic sign, signifier, structure, subject, psychoanalysis.

Fecha de recepción: octubre 30 de 2016

Fecha de aceptación: febrero 21 de 2017

Forma de citar (APA): Becerra, F. (2017). La noción de lenguaje en Jacques Lacan: Del Signo Lingüístico en Saussure al Algoritmo Saussureano en Lacan. Revista Filosofía UIS. 16 (1), doi: http://dx.doi.org/10.18273/revfil.v16n1-2017009

Forma de citar (Harvard): Becerra, F. (2017). La noción de lenguaje en Jacques Lacan: Del Signo Lingüístico en Saussure al Algoritmo Saussureano en Lacan. Revista Filosofía UIS. 16 (1), 179-192.

Fabián Becerra Fuquen: colombiano. Estudiante del doctorado en Psicología de la Universidad de Buenos Aires, Argentina. Profesor de la Universidad de Santander-UDES.

Correo electrónico: fabian.bfuquen@gmail.com

\footnotetext{
* Revisión de tema.
}

Revista Filosofía UIS, Vol. 16, No. 1, enero-junio de 2017 


\title{
LA NOCIÓN DE LENGUAJE EN JACQUES LACAN: DEL SIGNO LINGÜÍSTICO EN SAUSSURE AL ALGORITMO SAUSSUREANO EN LACAN
}

\begin{abstract}
Esperando que un mundo sea desenterrado por el lenguaje, alguien canta el lugar en que se forma el silencio. Luego comprobará que no porque se muestre furioso existe el mar, ni tampoco el mundo.

Por eso cada palabra dice lo que dice y además más y otra cosa.
\end{abstract}

Pizarnik, La palabra que sana.

\section{Introducción}

Es sin duda alguna la noción del lenguaje en Jacques Lacan un punto de entrada a sus fundamentos teóricos, lo que compete a su desarrollo sobre el lenguaje se encuentra en el transcurso de su obra como un elemento esencial, desde el principio hasta el final, sin embargo, se evidencia con mayor fuerza, particularmente, en el inicio y la mitad de su enseñanza, en donde introduce una de las tesis centrales sobre el inconsciente, la cual señala que el inconsciente está estructurado como un lenguaje, justamente porque "se resuelve por entero en un análisis del lenguaje, porque él mismo está estructurado como un lenguaje" (Lacan, 2011b, p.260).

Esta tesis propone el develamiento de la noción del inconsciente con la que Jacques Lacan inicia los desarrollos de su teoría, la cual quiere decir que el inconsciente tiene las mismas propiedades de estructura que las que tienen un lenguaje, por lo que cabe considerar la pregunta por las propiedades de estructura que tiene un lenguaje, ¿cuáles son dichas propiedades?

Para dar respuesta a tal pregunta se hace un Ilamado al Curso de Lingüística General de Ferdinand de Saussure (1970), a partir de la construcción del signo lingüístico, pues "llamamos signo a la combinación del concepto [significado] y de la imagen acústica [significante]" (p.129), que desempeña un papel esencial 
en la puesta en juego del lenguaje al nivel del grupo social, sin embargo, el signo lingüístico constituye la apertura del desarrollo acerca del lenguaje en Jacques Lacan, mediante la constitución de la primacía del significante a partir de lo que el psicoanalista francés denominó como el algoritmo saussureano, que a diferencia del signo lingüístico pone en juego el lenguaje al nivel del sujeto, esto es, a la mesura de su singularidad, a partir de la función que cumple el significante, pues es preciso concentrar la atención en las modificaciones que aporta Lacan al esquema de Saussure al representar su algoritmo, debido a que "el algoritmo saussureano del signo $\mathrm{S} / \mathrm{s}$ se incorpora al psicoanálisis y se transforma en una referencia permanente de la obra de Lacan" (Rabinovich, 2008, p.25).

Es así que el objetivo del presente texto consiste en el análisis de la noción del lenguaje en Jacques Lacan mediante el desarrollo del algoritmo saussureano con base en el fundamento teórico del signo lingüístico de Ferdinand de Saussure, esto permite al mismo tiempo constituir algunas consideraciones introductorias a los aportes teóricos hechos por Lacan, evidenciando el dialogo que este autor instaura con otras disciplinas, como es el caso de la lingüística de Saussure, y la manera en que este autor extrapola algunas nociones de esta disciplina para introducirlas al psicoanálisis, no sin antes darles nuevas significaciones.

\section{La lingüística de Ferdinand de Saussure: ¿̊lengua o lenguaje?}

Ferdinand de Saussure plantea los elementos pertinentes para poder determinar el objeto de estudio de la lingüística. Este es el principal logro de Saussure debido a que antes de él, la lingüística se ocupaba de otras cuestiones que obstaculizaban la posibilidad de fijar un único y verdadero objeto de estudio, y que por lo tanto impedía sentar sus bases en el campo de la ciencia.

Antes del logro de Saussure, la lingüística pasó por tres fases sucesivas:

Se comenzó por organizar lo que se llamaba la $<<$ gramática $>>$. Este estudio, inaugurado por los griegos, continuado principalmente por los franceses, está fundado en la lógica y desprovisto de toda visión científica y desinteresada de la lengua misma; lo que la gramática se propone únicamente es dar reglas para distinguir las formas correctas de las incorrectas; es una disciplina normativa, muy alejada de la pura observación, y su punto de vista es necesariamente estrecho.

Después apareció la filología [estudio de los textos escritos, su estructura y la evolución de una lengua y su desarrollo histórico y literario], (...) que quiere sobre todo fijar, interpretar, comentar los textos; (...) si la filología aborda cuestiones lingüísticas es sobre todo para comparar los textos de diferentes épocas, para determinar la lengua particular de cada autor, para descifrar y explicar inscripciones redactadas en una lengua arcaica u oscura; (...) pero, en ese terreno la crítica filológica falla en un punto: en que se atiene demasiado servilmente a la lengua escrita y olvida la lengua viviente. 
El tercer período comenzó cuando se descubrió que se podían comparar las lenguas entre sí. Este fue el origen de la filología comparativa o $<<$ gramática comparada $>>$. (...) consiste en aclarar una lengua por medio de otra, explicar las formas de una por las formas de las otras (Saussure, 1970, pp.39-40).

Lo que se evidencia es que en ninguna de estas fases se procuró determinar la naturaleza de su objeto de estudio, y sin tal delimitación, una disciplina es incapaz de encaminar un método, por consiguiente la pretensiones de establecer una ciencia que se constituya entorno a los hechos de lengua, debía esperar a que se pudiera comprender que la lengua no es una entidad, sino por el contrario la lengua viviente no existe más que en los sujetos hablantes.

Es así que la materia de la lingüística está constituida por todas las manifestaciones del lenguaje humano, ya se trate de pueblos salvajes o naciones civilizadas. Lo que hace justamente que la lingüística tenga conexiones estrechas con otras disciplinas como lo son la antropología, la sociología, la historia, la psicología, etc., lo que permite comprender que en la vida de los individuos y de las sociedades no hay factor tan importante como el lenguaje, por ello al lenguaje se lo puede tomar como un hecho social.

Sin embargo, aún no es claro żcuál es el objeto de estudio de la lingüística según Saussure? De entrada marca su posición advirtiendo: "hay que colocarse desde el primer momento en el terreno de la lengua y tomarla como norma de todas las otras manifestaciones del lenguaje" (Saussure, 1970, p.51). Esto evidencia que la lengua es parte fundamental del lenguaje, y no es el lenguaje mismo; al tiempo indica que lo primordial dentro del campo de la lingüística para Saussure, más que el lenguaje es la lengua, pues este último es su objeto de estudio, debido a que el lenguaje es multiforme y heteróclito, quiere decir, que evidencia diferentes dominios (físicos y psíquicos), pertenece al dominio individual y social, no se deja clasificar en ninguna de las categorías de los hechos humanos, y por tanto, dificulta la pretensión de ciencia en la lingüística.

Contrario al lenguaje, la lengua es susceptible de definición autónoma y de clasificación, y establece un orden diferente en la comprensión del lenguaje, ya no desde su dimensión diacrónica (histórica) sino desde su estatuto sincrónico, es decir, desde la lengua misma, permitiendo la rigurosidad en su estudio; esto no le resta importancia al lenguaje dentro de la teoría de Saussure, sin duda la lingüística esclarece elementos importantes sobre la facultad del lenguaje, pero no estudia directamente dicha facultad. 
Pero iqué es la lengua? La lengua no es más que una determinada parte del lenguaje, aunque esencial. Es a la vez un producto social de la facultad del lenguaje y un conjunto de convenciones necesarias adoptadas por el cuerpo social para permitir el ejercicio de esa facultad en los individuos. (Saussure, 1970, p.51).

Por consiguiente la lengua es adquirida y convencional, es un sistema de expresiones convencionales usados por una comunidad; es la parte social del lenguaje lo que lo hace exterior al individuo, en consecuencia el individuo solo no puede ni crearla ni modificarla; está determinada mediante criterios o normas implícitas de una sociedad; determina significados comunes, es decir, un sistema de signos, que permiten la comunicación y entendimiento, a partir del desarrollo cultural de tal comunidad, en un sentido presente (sincrónico); con ello se permite comprender la lengua como un sistema de signos.

Así como la lengua, el sistema de signos está determinado por un cuerpo social. Al respecto señala Saussure: "todo nos lleva a creer que por debajo del funcionamiento de los diversos órganos [aparato bocal, las áreas de broca, etc.] existe una facultad más general, la que gobierna los signos: esta sería la facultad lingüística por excelencia" (Saussure, 1970, p.53).

\section{El signo lingüistico}

Para comprender la lógica con la que actúa el signo lingüístico, cabría antes que nada determinar el lugar que ocupa la lengua en los hechos de lenguaje, a partir del circuito de la palabra:

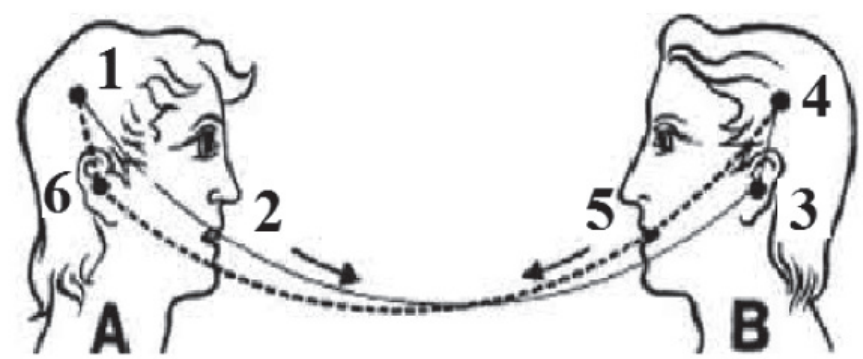

Tal circuito se lee siguiendo el orden ascendente en la numeración, siendo el punto 1 (uno) el lugar de partida, tal como se sigue a continuación:

1. En el cerebro del sujeto A se encuentran los hechos de consciencia, que reciben el nombre de conceptos, se hayan asociados con las representaciones o imágenes acústicas. Este es un fenómeno enteramente psíquico. 
2. Se sigue un proceso fisiológico, en donde el cerebro transmite a los órganos de la fonación un impulso correlativo a la imagen.

3. Luego, se haya un proceso físico que se pone en juego cuando las ondas sonoras se propagan de la boca de A al oído de B.

4. Una vez llegan las ondas sonoras al oído, llega la información al cerebro y se constituye en B el proceso de asociación entre el concepto e imagen acústica, generándose de nuevo el proceso, ahora en B.

5. Si B habla a su vez, este nuevo acto seguirá, a partir de las ondas sonoras emitidas por B.

6. Que serán recepcionadas por el oído de A. Y se continúa cuantas veces sean necesario el circuito.

Este proceso del circuito de la palabra, determina lo multiforme y heteróclito del lenguaje, implica como se evidencia aspectos tanto psíquicos, fisiológicos y físicos. Lo que le interesa a Saussure dentro de estas dimensiones del lenguaje es justamente ese aspecto que ha denominado como proceso psíquico, en el que se encuentra la relación o asociación entre el concepto y la imagen acústica, allí se puede localizar la lengua, lo que hace que la lengua sea un objeto bien definido en el conjunto heteróclito de los hechos de lenguaje, en ese punto en el que se asocia el concepto y la imagen acústica.

Precisamente, esta relación es la que obedece a los componentes que instituyen el signo lingüístico, tal como se observa en el siguiente gráfico:

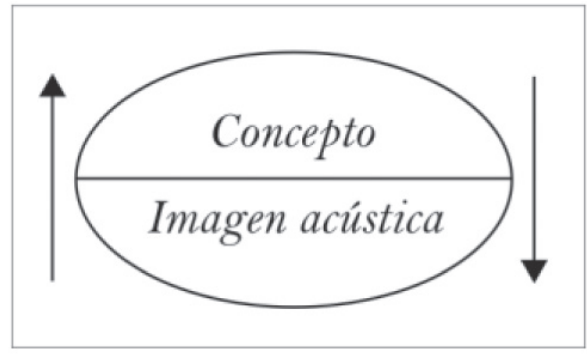

El signo lingüístico es la unidad básica de la lengua, el cual se comprende a partir de dos términos implicados, que están unidos por un vínculo de asociación.

Las flechas al costado, indican la relación reciproca que existe entre los dos elementos, ninguno se superpone al otro, tienen el mismo valor, y el círculo con la línea entre un elemento y otro representa unión entre los dos, pero "lo que el signo lingüístico une no es una cosa y un nombre, sino un concepto y una imagen acústica" (Saussure, 1970,p.128), es decir, que se trata de elementos con un carácter meramente psíquico, con una eficacia fundadora, que organiza el pensamiento. 
La imagen acústica no es el sonido material, cosa puramente física, sino su huella psíquica, es la representación que de él nos da el testimonio de nuestros sentidos; esa imagen es sensorial, y si llegamos a llamarla $<<$ material $>>$ es solamente en este sentido y por oposición al otro término de la asociación, el concepto, generalmente más abstracto (Saussure, 1945,p.128).

Es así que la imagen acústica no es otra cosa que la representación natural de la palabra, fuera de toda relación con el habla, un ejemplo de ello "aparece claramente cuando observamos nuestra lengua materna. Sin mover los labios ni la lengua, podemos hablarnos a nosotros mismos, recitarnos mentalmente un poema" (Saussure, 1970,p.28), por tal razón, la imagen acústica no es propiamente la voz proferida en relación al objeto, sino la representación de éste, creando una imagen del objeto, pero que por sí sola no indica mayor significación si no adviene junto a él un concepto, entonces, el concepto es la idea o significado que se tiene sobre algo, es decir, es el significado que determina el sentido sobre la imagen representada, esto es, sobre la imagen acústica; de tal manera que el signo lingüístico, se establece a partir de la relación intrínseca entre estos dos elementos, adquiriendo un vínculo recíproco, donde ninguno se sobrepone al otro y sí se comparten mutuamente, por consiguiente, uno no significa nada sin el otro.

Se muestra cómo la asociación entre la imagen acústica y el concepto indica un sentido de la percepción de la realidad. Simplemente con la falta de uno pierde todo sentido el otro. Por otra parte, Saussure le asigna de igual manera al concepto e imagen acústica los nombres de significado y significante respectivamente, para darle mayor comprensión a su estructura, pues si bien el concepto y la imagen acústica adquieren valor a través de su asociación, estos no son iguales, por consiguiente significado y significante son dos términos que señalan la oposición que los separa. Con todo, se observa el complejo sistema que rige la lengua, así como el dinamismo y estructura del lenguaje.
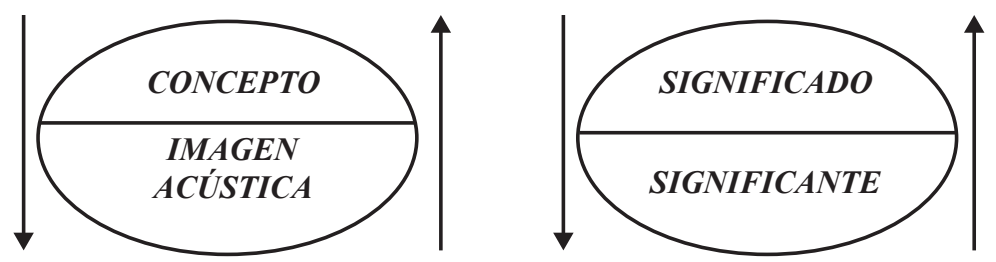

El signo (lingüístico) por sí solo no se sostiene, "un signo dado no existe sino por los otros signos. Más exactamente, un signo dado no existe sino por aquello que permite a los otros signos existir" (Milner, 2003, p.37). Un signo se sostiene en relación con los otros signos, permitiendo una relación de asociación no sólo entre los componentes internos de cada signo, sino con su relación externa entre signos, creando una cadena de signos. Estas relaciones - entre signos- son las que le dan valor y permiten la capacidad de constituir un pensamiento complejo. 


\section{La primacía del Significante: El algoritmo saussureano}

Ahora bien, ¿en qué punto entra la teoría de Jacques Lacan en este entramado sobre el signo lingüístico? Hay que retornar a la pregunta con la cual se inició este recorrido en la introducción del presente texto: ¿cuáles son aquellas propiedades de estructura que tiene un lenguaje? Con lo dicho hasta el momento se podría advertir que justamente se trata de una estructura de signos, sin embargo, vale agregar en palabras de Milner (2003):

La lingüística que interesa a Lacan es una lingüística que sostiene dos tesis: a) que se conocerá el lenguaje imponiéndose retener solamente de él las propiedades mínimas de un sistema cualquiera; pero también b) que sólo un sistema tiene propiedades. El nombre convenido del sistema cualquiera es justamente el de estructura (145).

Así la estructura entonces se presenta como un sistema cualquiera, el nombre de las propiedades cualquieras reducidas a sus propiedades mínimas es cadena. Se trata así de una estructura de cadena de signos. Sin embargo, a Jacques Lacan no le interesa tomar el signo en términos estrictamente lingüísticos tal como lo plantea Ferdinand de Saussure, en otras palabras, las propiedades mínimas de la estructura que le interesa a Lacan no es el signo lingüístico, sino uno de los elementos que se encuentran como propiedades mínimas del signo, es así que de la relación entre el significado y significante que se haya en el signo lingüístico, Lacan extrae al Significante 1 y lo define de la siguiente manera: "Un significante represente a un sujeto para otro significante" (Lacan, 2010a, p.799). A primera vista esta fórmula no arroja mayores luces, es necesario avanzar un poco para comprender la lógica del significante en Lacan.

La noción de estructura para Lacan (2013) es la de "un grupo de elementos que forman un conjunto co-variante. Dije un conjunto, no dije una totalidad" (pp.261-262). Con esto se refiere a que la estructura está constituida por estos elementos mínimos que son los significantes, y por su condición de estructura cada uno de estos elementos están articulados, al punto que si uno de estos varia, la estructura en su totalidad también lo hace, la estructura por esencia es interdependiente entre sus elementos, por tal razón su co-varianza.

\footnotetext{
1 A pesar que Lacan toma término significante de la lingüística no lo utiliza de la mismas manera como sucede en esta, ya que su valor en el campo de la lingüística se constituye en asociación con el significado, por lo tanto, al extraer el término de su campo natural e introducirlo al campo del psicoanálisis, pierde el sentido propiamente lingüístico; intentar mantener su noción bajo tales condiciones llevaría a un forzamiento del término, conduciéndolo a decir lo que quizá, el término en sí mismo, no pueda decir. Por esta razón al introducir el término en la estructura teórica del psicoanálisis, la palabra significante adquiere un sentido nuevo, pues ahora de lo que se trata es de la primacía del significante.
} 


\section{Ahora bien:}

La categoría conjunto, para introducirla, encuentra nuestro acuerdo, por cuanto evita las implicaciones de la totalidad o las depura. Pero esto no es para decir que sus elementos no sean aislables, ni sumables: por lo menos, si buscamos en la noción de conjunto alguna garantía del rigor que tiene en la teoría matemática" (Lacan, 2010b, p.618).

Esta estructura no obedece a un orden cerrado, no plantea una totalidad, de allí que la defina en términos de un conjunto abierto que permite un dinamismo gracias a que hay un elemento que falta ${ }^{2}$, que es imposible de significar, un real dice Lacan, y con base en este real se arma la estructura de los significantes, y esta estructura no es otra que la del sujeto. Se trata de un sujeto analítico, no es el sujeto en su totalidad. "Si fuéramos totales, cada uno sería total por su lado y no estaríamos aquí, juntos, tratando de organizarnos" (Lacan, 2012, p.365).

Que se trate de una estructura sin una totalidad, obedece al orden lógico del inconsciente, que contradice la pretensión científica de la certeza y la unificación de los sujetos, desatendiendo lo que le compete a la particularidad del deseo del sujeto. Por esta razón el interés de plantear la supremacía del significante, como vía que permite constituir una ética que se sostiene en la posibilidad de tomar al sujeto por su singularidad, esto es, definirlo por su diferencia de los otros sujetos.

Ahora, los significantes le son dados al sujeto por su relación con el Otro $(\mathrm{A})^{3}$, entendiendo al Otro como el tesoro de los significantes, el lugar desde donde le vienen los significantes que definen al sujeto. El Otro es esencialmente un lugar simbólico, pues es en la existencia del Otro que se funda al sujeto. "El Otro es precisamente el lugar del tesoro, digamos, de aquellas frases, incluso de aquellos prejuicios sin los cuales la agudeza no pude cobrar valor y fuerza" (Lacan, 2010c, p.121). El Otro es tanto alguien como algo (padre, madre, cultura, etc.) que ha ocupado un lugar determinante para la existencia del sujeto, por tal razón, el campo del significante es el campo del Otro.

Entonces, de lo que trata en Lacan es de la primacía del significante, que se evidencia a partir del algoritmo saussureano:

\footnotetext{
${ }^{2}$ Una forma de imaginar una estructura dinámica constituida por una falta, es la del Puzzle deslizante, la estructura de este juego cobra un dinamismo gracias a que hay una pieza que falta, dicha pieza cobra valor justamente por su ausencia. De lo contrario se trataría de una estructura fija.

${ }^{3}$ El Otro es representado por la letra "A" mayúscula, dado que en francés Otro se escribe Autre, Lacan toma la primera letra del Autre, en oposición al autre (a), este otro con "a" minúscula es el otro semejante, entendido no como un lugar simbólico sino por el contrario es imaginario.
} 


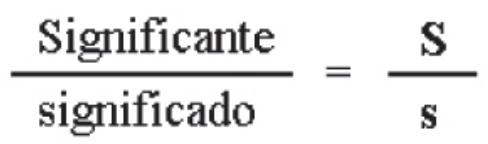

Lo llama saussureano en reconocimiento a Saussure, y se lee así: "significante sobre significado, el sobre responde a la barra que separa sus dos pisos" (Lacan, 2011a, p.464). Para Lacan no se trata de una unión entre el significante y significado, justamente por ello su algoritmo no contiene las flechas ni el círculo que representaba la unión entre sus dos elementos tal como se observaba en el signo lingüístico, por el contrario estos elementos se constituyen como órdenes distintos separados por la barra resistente a la significación. Esto hace que se subraye la primacía del significante el cual aparece en la parte superior del algoritmo representado por la letra mayúscula: $S$, cuya determinación estaría dada en la posibilidad de traspasar la barra que impide se produzca un significado, por lo que el significado representado por la letra minúscula: s, aparece como una incógnita, como un elemento a descifrar. Podría representarse con la siguiente fórmula:

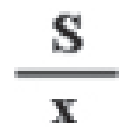

En donde la solución del algoritmo se estipula con la posibilidad de determinar un significado (s), se trata de esclarecer el valor de la " $\mathrm{x}$ " como representante del significado desconocido, y el valor algorítmico consiste en que el significado es efecto de la operación significante, con la dificultad que plantea la barra imposibilitando su atravesamiento. Por lo que se trata de una pura función significante.

Lacan sostiene que hay un orden de significantes puros en el que los significantes existen antes de los significados (Evans, 1997, p.178), estos significantes puros, son significantes sin significado; ejemplo de ello, podría ser cuando un niño a muy temprana edad observa el coito de sus padres (S1) y no tiene un referente significativo dentro de esa cadena de significantes (S2), dada su corta edad no tiene con que proporcionar sentido alguno a tal evento, por tanto ese significante primero (S1) no significa nada porque no hay una batería de significantes (S2) en la que se articule para determinar un significado, pero a posteriori, este significante se va ir articulando en el discurso del sujeto, en la medida que éste pueda hablar sobre los acontecimientos de su historia y darle alguna significación. Hay que recordar que el sujeto resignifica su historia mediante su discurso en un psicoanálisis, articulando los significantes que lo definen. 
De allí que Lacan (2013) en El Seminario 3: Las Psicosis, subraye que "Un significante, en cuanto tal, no significa nada" (p.261). Esto es, que se necesitan al menos dos significantes que se articulen para que pueda atravesarse la barra, pues uno en cuento tal no alcanza; los significantes deben articularse en cadena, para constituir una estructura cuya propiedad está determinada por la cadena de significantes. Pues el algoritmo, en cuanto que el mismo no es sino pura función del significante, no puede revelar sino una estructura de significante. "Esto es lo que hará posible un estudio exacto de los lazos propios del significante y de la amplitud de su función en la génesis del significado" (Lacan, 2011a, p.465).

Es así que la estructura está compuesta por la correlación entre significantes diferentes, pues el significante responde a una estructura diferencial, ya que su reconocimiento se da por tal diferencia, cada significante se define por oposición a los demás dentro de una cadena, estando entonces, la cadena conformada por la articulación entre significantes diferentes:

\section{$\mathrm{S} 1 \Rightarrow \mathrm{S} 2$}

Esto le permite retomar la noción de significante y hacer mayor énfasis en el el resultado algorítmico de dicha relación significante, es decir, en el sujeto:

Nuestra definición del significante (no hay otra) es: un significante es lo que representa al sujeto para otro significante. Este significante será pues el significante para el cual todos los otros significantes representan al sujeto: es decir que a falta de este significante, todos los otros no representarían nada (Lacan, 2010d, p.779).

El sujeto aparece en entre el vínculo significante, pues el significante tiene esta cualidad de representar un sujeto para otro significante, en oposición al signo lingüístico de Saussure, pues "cada signo es, como dice Lacan, lo que representa algo para alguien" (López, 2009, p.28).

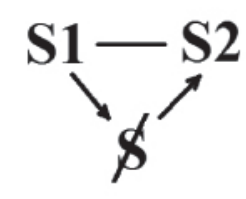

Esto señala el efecto del lazo significante, en la medida que la articulación entre estos permite alcanzar la determinación significante, esto es, la posibilidad de traspasar la barra que impide se produzca un significado y por consiguiente aparece el desciframiento de la incógnita alojada en algoritmo; en el lugar de la " $x$ " emerge como efecto un sujeto, un sujeto que trae consigo una verdad 
inconsciente. Este es el enigma a descifrar, el lugar que el sujeto ocupa en su articulación significante. Pues se trata del sujeto del inconsciente, y el significante está íntimamente ligado a él, por esta razón la definición del significante es la misma definición del sujeto, siendo este quien está representado por un significante para otro significante.

\section{A modo de cierre}

Esta particularidad del significante a diferencia del signo, hace que Lacan señale la singularidad del sujeto, esto es, que mientras el signo lingüístico en Saussure plantea una construcción dada por convención (común acuerdo) en un grupo social, en el cual se pueda determinar el sentido del signo, como siendo lo que representa algo para alguien dentro de ese grupo social, por el contrario, Lacan se dirige al caso por caso, a la singularidad que define al sujeto y su modo de padecer, que obliga al psicoanalista direccionar al analizante (paciente) hacia sus propios significantes que en articulación señalan el significado de su verdad inconsciente.

Esto quiere decir, que el psicoanalista debe despojarse de todo saber, de todo sentido previo frente al discurso del analizante, trabajar con el significante lo obliga a tomar una posición similar a la de la docta ignorancia cuyo objetivo busca significar la actitud prudente del sabio ante la dimensión de los problemas que encausan la existencia del ser. Por esta razón, si un paciente llega a una terapia psicoanalítica diciendo 'Soy virgen', señalando éste como el motivo de su consulta, el psicoanalista no puede interpretar anticipadamente que lo que demanda el paciente es la pérdida de su virginidad como solución definitiva; sabrá que éste hecho en sí no es el problema, sino que el malestar está en el centro de lo que esto -ser virgen- podría llegar a representar para el sujeto; se le debe preguntar sobre dicha condición y solicitarle que asocie libremente para ver su articulación con otros significantes. Para ello el analista tendrá que despojarse del prejuicio y del saber anticipado, pues comprende que el saber que proviene del inconsciente está del lado del sujeto, aunque éste último no tenga la plena consciencia de ello; quien mejor que el paciente para evidenciar un saber sobre su propio sufrimiento, sin embargo, hay que darle la palabra para que pueda hallar algunos despojos sobre dicho saber inconsciente.

A pesar que el sujeto contiene la verdad sobre su padecer seguramente la desconoce, y es por la esencia de su estructura constituida por la lógica del significante que logra advertir justamente algo de dicha verdad sobre su padecer inconsciente. Es por el lazo significante que es posible develar la singularidad del sujeto del inconsciente. De allí que el inconsciente este estructurado como un lenguaje, pues este lenguaje como se ha dicho es de estructura de significantes y el inconsciente surge allí entre significantes para hablar sobre la particularidad del sujeto. Esto abre la vía del caso por caso en la clínica psicoanalítica, de la puesta 
en juego de la subjetividad como lo más propio a cada uno y que se define, como el significante, por oposición a los demás, de allí que Lacan (2009) afirme que "el psicoanálisis es una experiencia de lo particular" (40) $\varphi$

\section{REFERENCIAS}

Evans, D. (1997). Diccionario Introductorio de Psicoanálisis Lacaniano. Buenos Aires, Bueno Aires: Paidós.

Lacan, J. (2009). El Seminario, libro 1. Los Escritos Técnicos de Freud. Buenos Aires: Paidós.

Lacan, J. (2010a). Posición del inconsciente. En Escritos 2. Buenos Aires: Siglo Veintiuno editores.

Lacan, J. (2010b). Observación sobre el informe de Daniel Lagache: Psicoanálisis y estructura de la personalidad. En Escritos 2. Buenos Aires: Siglo Veintiuno editores.

Lacan, J. (2010c). El Seminario, libro 5. Las Formaciones del Inconsciente. Buenos Aires: Paidós.

Lacan, J. (2010d). Subversión del sujeto y dialéctica del deseo en el inconsciente freudiano. En Escritos 2. Buenos Aires: Siglo Veintiuno editores.

Lacan, J. (2011a). La instancia de la Letra en el inconsciente, o la razón desde Freud. En: Escritos 1. Buenos Aires: Siglo Veintiuno editores.

Lacan, J. (2011b). Función y campo de la palabra y del lenguaje en psicoanálisis. En Escritos 1. Buenos Aires: Siglo Veintiuno editores.

Lacan, J. (2012). El Seminario, libro 2. El Yo en la teoría de Freud y en la Técnica Psicoanalítica. Buenos Aires: Paidós.

Lacan, J. (2013). El Seminario, libro 3. Las psicosis. Buenos Aires: Paidós.

López, H. (2009). La instancia de Lacan: actualidad de la instancia de la letra en el inconsciente o la razón desde Freud. Mar del Plata: EUDEM.

Milner, J. (2003). El periplo estructural: Figuras y paradigma. Buenos Aires: Amorrortu.

Pizarnik, A. (2014). Poesía Completa. Barcelona: Lumen

Rabinovich, D. (2008). Sexualidad y significante. Buenos Aires: Ediciones Manantial.

Saussure, F. (1970). Curso de lingüística general. Buenos Aires: Editorial Losada. 